\title{
Numerical Analysis of Cooling Down a Micro ATX Computer Chassis
}

\author{
Tolga DEMIRCAN \\ Kırlkkale University, Mechanical Engineering Department, Kırlkkale, 71450, Turkey, E-mail: tolgademircan@gmail.com
}

cross $^{\text {ref }}$ http://dx.doi.org/10.5755/j01.mech.24.5.19233

\section{Introduction}

In the last century, science and technology is constantly evolving. The building stone of this evolution is the development of computer technologies. The reason for acceleration in computer development is linked with all technological developments. For this reason, researches were directed to develop computers with higher operating speed and lower volumes. As the computer chasses has smaller volumes, this revealed the need for electronic circuits that work with higher loads. As the volume decreased, heat per unit volume has increased and maintaining constant operating temperatures became a significant challenge. Researches have worked on cooling electronic circuits inside the computer as well as safe operating conditions for the systems.

Nikas and Panagiotou [1] numerically evaluated heat transfer and flow characteristics inside a computer chassis using Fluent package program. The authors stated that it is possible to provide optimum temperature in the chassis with passive cooling. The results of the study showed that a large fan with constant flow rate was more effective rather than two small fans. Chang et al. [2] investigated cooling a desktop computer with minimum air flow using computational fluid dynamics. For this purpose, the authors made simulations with a CPU with $30 \mathrm{~W}$ power consumption. The result of the design indicated that the processor temperature can be decreased until $62,9 \mathrm{C}^{\circ}$. Kattekola [3] analysed thermal characteristics of a tablet computer and the main components of the tablet using experimental and numerical methods. Numerical and experimental data were compliant. Approximately $4 \mathrm{C}^{\circ}$ temperature difference is observed in the chassis, and approximately $2 \mathrm{C}^{\circ}$ temperature difference is observed on the surfaces.

Yu and Webb [4] numerically analysed the thermal characteristic of a desktop computer using Icepak package program. Processor of a computer model with $80 \mathrm{~W}$ power consumption was investigated in terms of cooling. According to the chassis design, the temperature of the chassis and the processor were within the acceptable region. Saini and Webb [5] investigated air cooling limits of heat exchanger with plate paddles used for cooling the computer. The results of the study show that as the fan velocity of the system is increased by $25 \%$ and the surface area of heat sink is increased $33 \%$, heat sink has reached to $103.4 \mathrm{~W}$ cooling limit. Ozturk and Tari [6] numerically analysed three different heat sink model for cooling. The geometry and material of the heat sink is changed and these changes are analysed using Fluent and Icepak package programs. The results indicated that copper material had increased the cooling performance. Murugan et al [7] investigated thermal performance of all the electronic components inside a personal desktop computer using Ansys Fluent package program. The best system performance in the model was observed when the fan input velocity is between $1.5-2 \mathrm{~m} / \mathrm{s}$.

Anandakrishnan and Balaji [8] investigated flow structure and thermal behaviour inside a desktop computer with multiple cores using computational fluid dynamics. The results determined that processor temperature and power dissipation had linear relationship yet the fan velocity was non-linear relationship with processor temperature. Temperature of other electronic components inside the system had increased with the increase in thermal dissipation of the processor. The study determined that the ambient air transferred inside the chassis has a significant effect on CPU and chassis temperature. Kim et al. [9] experimented on cooling the processor of a desktop computer using thermal tubes. Cooling with thermal tube had relatively better cooling performance compared to fan systems under $2950 \mathrm{rpm}$. Thermal tube has a lower noise and higher cooling performance compared to cooling with fan system.

Ren [10] designed a chassis with electronic components using Icepak package program. The analyses indicated that the chassis with $80 \mathrm{~W}$ power consumption was adequately cooled using forced convection. Chian et al. [11] analysed heat transfer on a desktop computer with heat generating components using PHOENICS numerical code. The simulations are conducted for different fan locations on both sides of the computer chassis and for different Reynolds values. The results show that locations of the fans and Reynolds values had significant effect on thermal transfer. Hunasikatti et al. [CPU] analysed flow and thermal properties of a chassis with motherboard and hard disk using Fluency package program. The study focused on temperature distribution on the motherboard which is homogenously heated. There different models are analysed in the study; vertical CPU position, horizontal CPU position, and chassis with exhaust fans. When CPU is positioned horizontally, the temperature of motherboard relatively decreased compared to vertical position of CPU. Xu et al. [13] numerically analysed flow and thermal distribution inside a single board computer chassis using FloEFD software. The computer chassis is evaluated under air cooling and liquid cooling conditions. The result of the study showed that liquid cooling can provide adequate cooling for all the electronical equipment inside the system. When the system is cooled with liquid, CPU temperature decreased 56\% compared to cooling with air. Mohan and Govindarajan [14] numerically calculated thermal analysis of cooling a computer chassis using computational fluid dynamics. For this purpose, the effects of heat sink with different paddle geometry on flow and thermal characteristic were investigated. The paddle geometry for optimum cooling is determined for the computer chassis.

When the literature is reviews, there are various studies on cooling the computer components. However, 
these studies focused on CPU, motherboard, and other computer components separately. There are few examples that investigate the performance of all the components in the system. For this purpose, in this study Micro ATX computer chassis geometry is designed and cooling the computer chassis with fans is numerically modelled using Computational Fluid Dynamics. Different fan revolutions are calculated to analyse the heat under idle and full-load operations. The simulation results are used for evaluating velocity and temperature distributions in the solution region and operation parameters for optimum cooling are determined.

\section{Definition of the problem and mathematical formulation}

In this study, effect of fan flows on micro ATX chassis cooling is investigated. The chassis shown in Fig. 1 is designed for analyses. The chassis includes motherboard, processor (CPU), graphic card, power supply, DVD driver, RAM, hard disk (HDD), and fan. Temperature values are defined for idle and full-load conditions and a model is generated. This model is simulated using computational fluids dynamics (CFD) package program for different velocity parameters and velocity and temperature distributions are obtained for solution region of the problem.

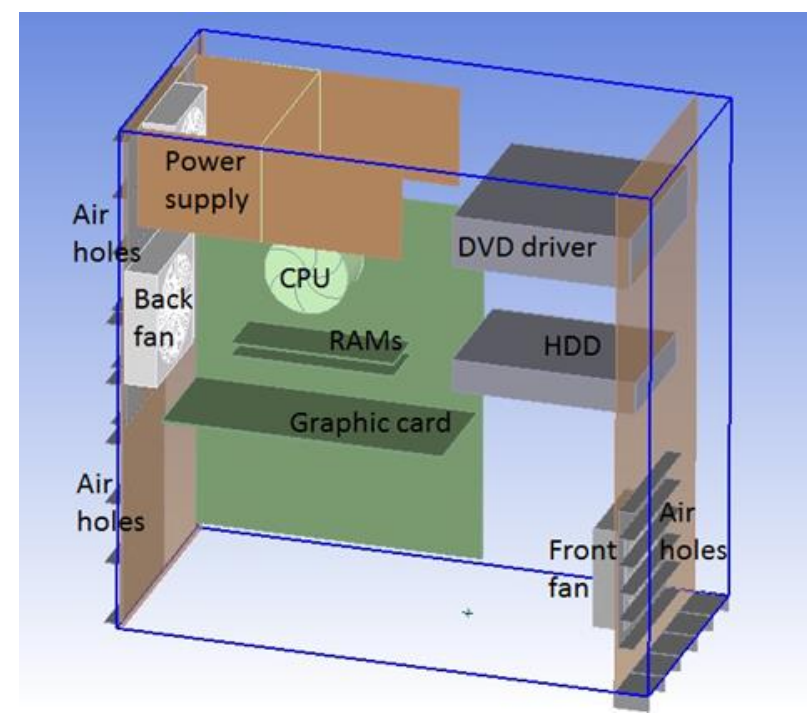

Fig. 1 Schematically representation of the problem

The outer dimensions of the micro ATX chassis are 310x410x660 mm. Other main computer component dimensions and locations are determined according to literature review. k- $\varepsilon$ turbulence approach is adopted for turbulence. For idle and full-load operations, simulation is repeated for 1000, 2000, and $3000 \mathrm{rpm}$ fan revolutions. Continuity, energy, momentum, and turbulence equations of the problem are stated below.

\subsection{Differential equations of problem}

Continuity Equation:

$$
\frac{\partial \bar{u}_{j}}{\partial x_{j}}=0
$$

$$
\begin{aligned}
& \frac{\partial}{\partial x_{j}}\left(\bar{u}_{i} \bar{u}_{j}\right)=f_{i}-\frac{1}{\rho} \frac{\partial \bar{p}}{\partial x_{i}}+\frac{\partial}{\partial x_{j}}\left[\frac{\mu}{\rho}\left(\frac{\partial \bar{u}_{i}}{\partial x_{j}}+\frac{\partial \bar{u}_{j}}{\partial x_{i}}\right)\right]+ \\
& +\frac{1}{\rho} \frac{\partial}{\partial x_{j}}\left(-\rho \overline{u_{i}^{\prime}} \overline{u_{j}^{\prime}}\right) .
\end{aligned}
$$

Energy Equation:

$$
\frac{\partial}{\partial x_{j}}\left(\bar{u}_{i} \bar{T}\right)=\frac{\partial}{\partial x_{j}}\left[\alpha\left(\frac{\partial \bar{T}}{\partial x_{j}}\right)\right]+\frac{1}{\rho c_{p}} \frac{\partial}{\partial x_{j}}\left(-\rho c_{p} \overline{u_{j}^{\prime}} \overline{T^{\prime}}\right)
$$

Turbulence $k-\varepsilon$ Equations:

$$
\begin{aligned}
& \frac{\partial}{\partial x_{j}}\left(\rho k u_{j}\right)=\frac{\partial}{\partial x_{j}}\left[\left(\mu+\frac{\mu_{t}}{\sigma_{k}}\right) \frac{\partial k}{\partial x_{j}}\right]+G_{k}-\rho \varepsilon+S_{k}, \\
& \frac{\partial}{\partial x_{j}}\left(\rho \varepsilon u_{j}\right)=\frac{\partial}{\partial x_{j}}\left[\left(\mu+\frac{\mu_{t}}{\sigma_{\varepsilon}}\right) \frac{\partial \varepsilon}{\partial x_{j}}\right]+ \\
& +C_{1 \varepsilon} \frac{\varepsilon}{k} G_{k}-C_{2 \varepsilon} \rho \frac{\varepsilon^{2}}{k}-S_{\varepsilon} .
\end{aligned}
$$

Turbulence viscosity:

$$
\mu_{t}=\rho C_{\mu} \frac{k^{2}}{\varepsilon}
$$

The constants used in this model are taken as follows [15]: $C_{1 \varepsilon}=1.44, C_{2 \varepsilon}=1.92, C_{\mu}=0.09, \sigma_{k}=1.0$ and $\sigma_{\varepsilon}=1.3$.

\subsection{Boundary conditions}

Two fans, back and front fan, are mounted on the computer chassis. Front fan provides suction towards the chassis and back fan blows the air inside the chassis. Revolution of each fan is kept constant and same in each simulation. Air velocity transferred by fans and fan revolution changes are indicated in Table 1.

Table 1

Back and front fan air velocities

\begin{tabular}{|c|c|c|}
\hline $\begin{array}{c}\text { Fan rev } \\
(\mathrm{rpm})\end{array}$ & $\begin{array}{c}\text { Front fan air vel. } \\
(\mathrm{m} / \mathrm{s})\end{array}$ & $\begin{array}{c}\text { Back fan air vel. } \\
(\mathrm{m} / \mathrm{s})\end{array}$ \\
\hline 1000 & 3.77 & 4.34 \\
\hline 2000 & 7.54 & 3.68 \\
\hline 3000 & 11.31 & 13.00 \\
\hline
\end{tabular}

Table 2

Power consumption of main components

\begin{tabular}{|l|c|c|}
\hline Component name & Full-load & Idle \\
\hline Processor & $70 \mathrm{~W}$ & $48 \mathrm{~W}$ \\
\hline Graphic card & $40 \mathrm{~W}$ & $18 \mathrm{~W}$ \\
\hline Power supply & $60 \mathrm{~W}$ & $35 \mathrm{~W}$ \\
\hline HDD & $15 \mathrm{~W}$ & $5 \mathrm{~W}$ \\
\hline DVD driver & $5 \mathrm{~W}$ & $0 \mathrm{~W}$ \\
\hline RAMs & $10 \mathrm{~W}$ & $8 \mathrm{~W}$ \\
\hline
\end{tabular}

Momentum Equations: 


\subsection{Verification of the numerical method}

The problem in this study is solved numerically under appropriate boundary conditions using computational fluid dynamics. To control the reliability and accuracy of the numerical results of the simulations, test analyses is conducted using the geometry and boundary conditions of a similar study on the literature [8]. These test results and reference results in the related study are compared. Fig. 2 shows temperature values for 2850 and $5000 \mathrm{rpm}$ fan revolutions in both operations.

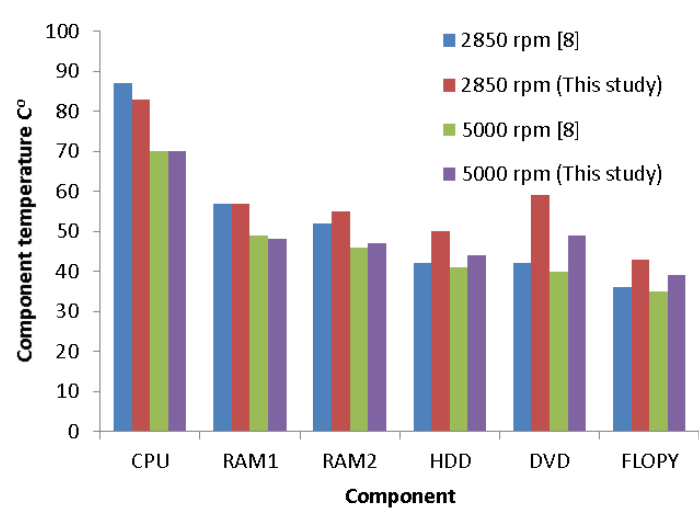

Fig. 2 Comparison of the current results with literature

\section{Results and evaluation}

In this study, effect of fan flows on micro ATX chassis cooling is investigated. For this purpose, the chassis was designed including motherboard, processor (CPU), graphic card, power supply, DVD driver, RAM, hard disk (HDD), and fan. Power consumptions of these components are defined for idle and full-load operations and simulated for 1000-3000 rpm fan revolutions. After the simulations, maximum temperatures for each component are determined using velocity and temperature distributions in the solution region and the results are evaluated below.

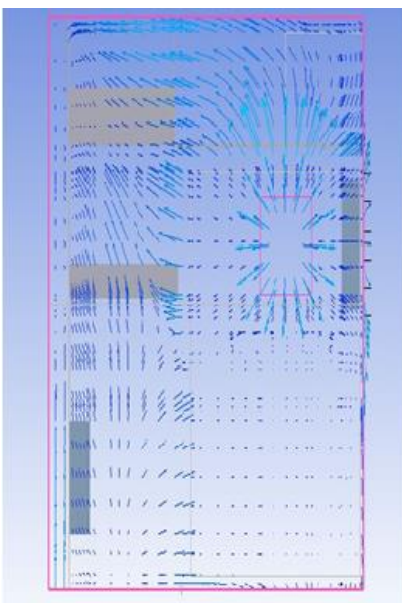

a

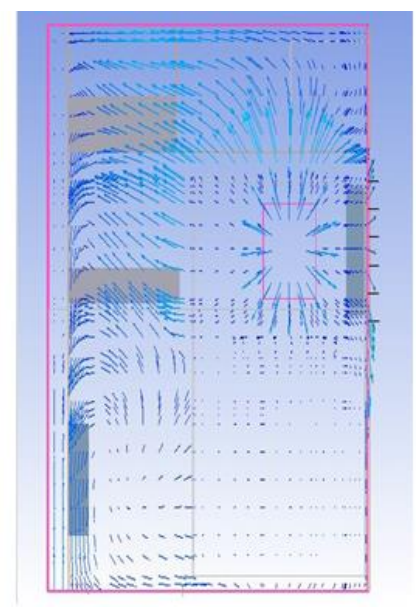

b
Fig. 3 Velocity vector distributions for different fan revolutions a) $1000 \mathrm{rpm}$, b) $3000 \mathrm{rpm}$

Fig. 3 shows velocity vector distributions for different fan revolutions on the surface selected behind the processor. As the figure indicates, vector distributions for both revolutions have similar structures. In general, air absorbed from front fan on the front surface is distributed inside the chassis, the system components are cooled as the air contacts, and directed to the back surface to be exhausted by the back fan. Certain portion of the air is exhausted from the air output holes. Fundamentally, main computer components inside the chassis effect the air flow, changes the direction and velocity of the air, and creates circulation regions inside the chassis. The figures show that as the fan flow increases, magnitude of the velocity vectors increases. Yet, the location of the circulation regions and general flow structure are constant.

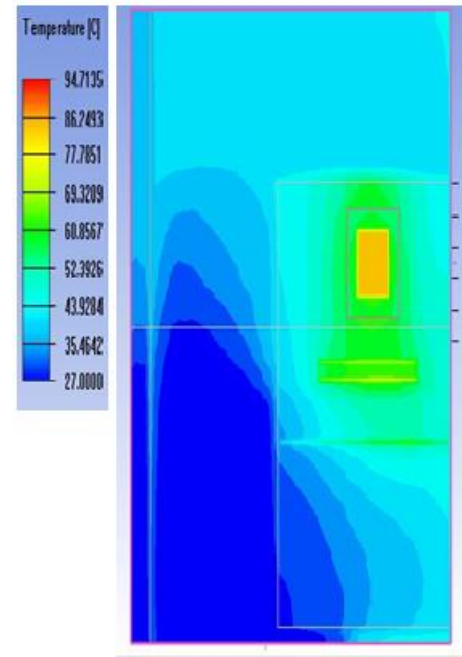

a

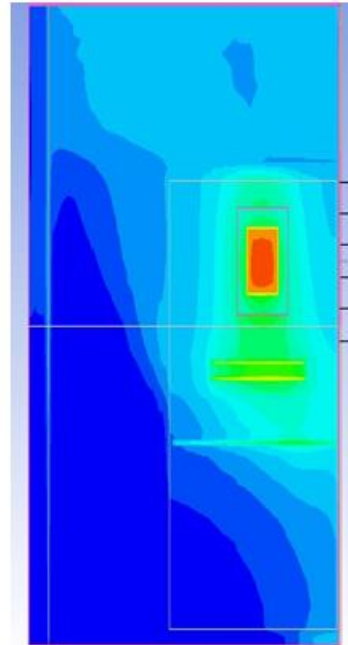

b
Fig. 4 Temperature contours for $1000 \mathrm{rpm}$ fan revolution a) idle operation, b) full-load operation

In Fig. 4, temperature contours for idle and fullload operations under $1000 \mathrm{rpm}$ fan revolution are given for a surface at the back of the processor. As the figure shows, for both conditions, processor regions are subjected to high temperatures. Especially, when the computer is operating under full-load, the power consumption of CPU is higher, thus, CPU and the surrounding components have higher temperature regions. Generally, air circulation in this revolution is insufficient to cool the components in the chassis both in idle operation and full-load operation.

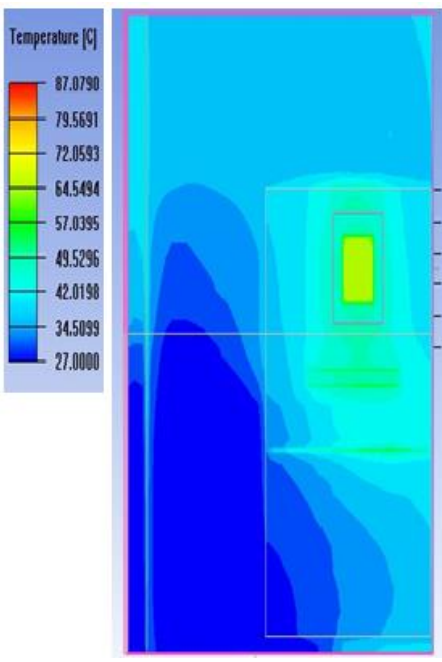

a

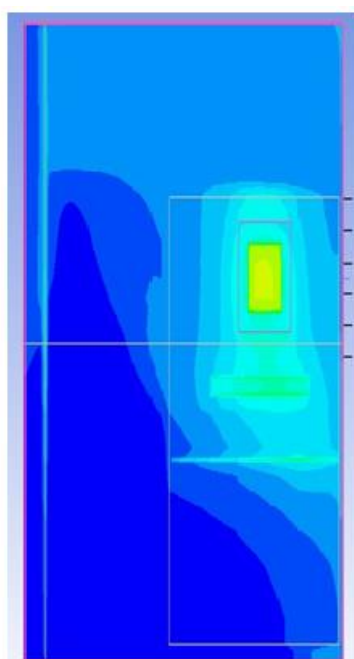

$\mathrm{b}$
Fig. 5 Temperature contours for $2000 \mathrm{rpm}$ fan revolution a) idle operation, b) full-load operation 
In Fig. 5, temperature contours for idle and fullload operations under $2000 \mathrm{rpm}$ fan revolution are given for a surface at the back of the processor. The figure indicates that increased fan revolution and air flow has insignificant effect on general structure of temperature contours inside the chassis. Yet, when CPU is analysed, temperature values are relatively lower. Thus, as fan flow is increased, effects of high temperature regions on the sensitive regions inside the chassis are decreased. Especially, under idle operation, safe operating temperatures are observed for the processor and other components. Yet, under full-load operation, there are high temperature regions around the processor and the cooling is insufficient.

Fig. 6 shows the change in maximum temperature values observed on the computer main components under idle operation as the fan revolution changes. The figure shows that the main components analysed in this study such as CPU, Power supply, HDD, DVD driver, RAM 1, and RAM 2 has maximum temperature. To ensure a safe operating temperature, the main components inside the chassis should be below $80^{\circ} \mathrm{C}$ operating temperature. The figure shows that when the system is under idle operation and for $1000 \mathrm{rpm}$ fan revolution, cooling is sufficient for components other than power supply and RAM1. As the fan revolution increases, the temperatures of these components are inside the safe operating temperatures. Therefore, when the system is under idle operation and has $2000 \mathrm{rpm}$ or higher revolutions, the cooling will be sufficient for all system.

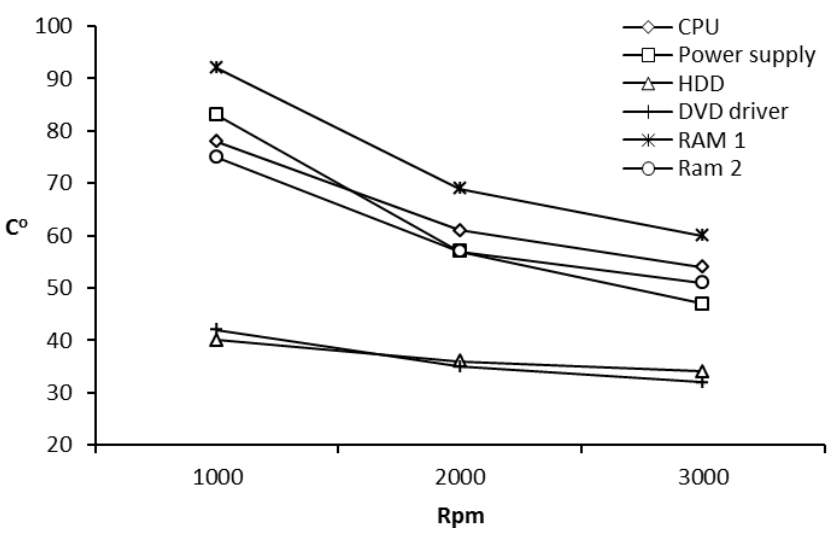

Fig. 6 Change in system main components with fan revolution under idle operation

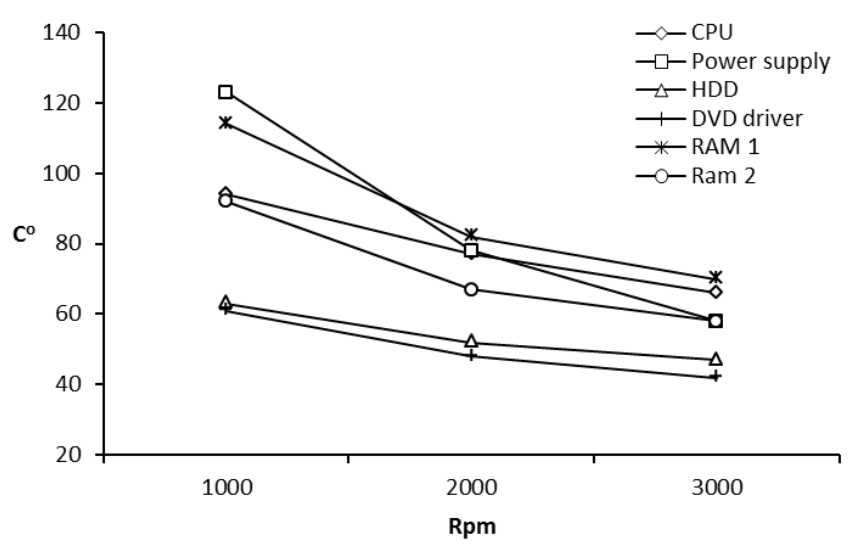

Fig. 7 Change in system main components with fan revolution under full-load operation

In Fig. 7, under full-load operation, change in system main components with fan revolutions is indicated. The figure shows that under full-load operation, the temperature of all components increases, therefore each component has higher temperatures. Especially, flow created with 1000 and $2000 \mathrm{rpm}$ fan revolution is insufficient to cool the system components. As the fluid flow is increased, forced convection increases and the component temperatures decrease. When fan revolution is $3000 \mathrm{rpm}$, flow rate is effective for cooling. For $3000 \mathrm{rpm}$ fan revolution, all system components are below $80 \mathrm{C}^{\circ}$ which is considered as safe operating temperature.

\section{Conclusions}

In this study, effect of fan flows on micro ATX chassis cooling is investigated. For this purpose, the chassis was designed including motherboard, processor (CPU), graphic card, power supply, DVD driver, RAM, hard disk (HDD), and fan. Power consumptions of these components are defined for idle and full-load operations and simulated for 1000-3000 rpm fan revolutions. The simulation results show that when the computer is under idle operation, main components of the system have relatively lower temperatures and low fan revolutions are sufficient for adequate cooling. However, when the computer is under full-load operation, cooling the chassis is challenging and lower fan revolutions are insufficient. Therefore, high fan flows are effective to keep the system inside the safe operating temperature region as the convection heat transfer inside the chassis increases.

\section{References}

1. Nikas, K. S. P.; Panagiotou, A. D. 2013. Numerical Investigation of Conjugate Heat Transfer in a Computer Chassis, Journal of Advanced Mechanical Engineering 1: 40-57. https://doi.org/10.7726/jame.2013.1004.

2. Chang, J. Y.; Yu, C. W.; Webb, R. L. 2001. Identification of minimum air flow design for a desktop computer using CFD modeling, Journal of Electronic Packaging 23: 225-231. https://doi.org/10.1115/1.1348012.

3. Kattekola, R. 2008. Numerical analysis of thermal characteristics of a tablet computer and its internal components, Master of Science, Department of Mechanical Engineering of the College of Engineering and Applied Science.

4. Yu, C. W.; Webb, R. L. Thermal design of a desktop computer system using CFD analysis, Seventeenth IEEE SEMI-THERM Symposium, 18-26. https://doi.org/10.1109/STHERM.2001.915139.

5. Saini, M.; Webb, R. L. 2003. Heat rejection limits of air cooled plane fin heat sinks for computer cooling, IEEE Transactions on Components and Packaging Technologies 26(1): 71-79. https://doi.org/10.1109/TCAPT.2003.811465.

6. Ozturk, E.; Tari, I. 2008. Forced air cooling of CPUs with heat sinks: A Numerical Study, IEEE Transactions on Components and Packaging Technologies 31(3): 650-660. https://doi.org/10.1109/TCAPT.2008.2001840.

7. Murugan, P. C.; Fathimasurumy, S.; Joseph Sekhar, S. 2016. Numerical studies on the electronic gadgets in 
a personal computer using CFD, International Journal of Science and Research 5(7): 1508-1511. https://doi.org/10.21275/v5i7.ART2016526.

8. Anandakrishnan, M.; Balaji, C. 2009. CFD simulations of thermal and flow fields inside a desktop personal computer cabin with multicore pProcessors, Engineering Applications of Computational Fluid Mechanics 3(2): 277-288. https://doi.org/10.1080/19942060.2009.11015271.

9. Kim, K. S.; Won, M. H.; Kim, J. W.; Back, B. J. 2003. Heat pipe cooling technology for desktop PC CPU, Applied Thermal Engineering 23: 1137-1144. https://doi.org/10.1016/S1359-4311(03)00044-9.

10. Ren, H. 2015. Thermal design of a cabinet with heat sinks using CFD analysis, International Journal of Science, Engineering and Technology Research 4(7): 23462350 .

11. Chiang, J. S.; Chuang, S. H.; Wu, Y. K.; Lee, H. J. 2005. Numerical simulation of heat transfers in a desktop computer with heat-generating components, International Communications in Heat and Mass Transfer 32: 184-191.

https://doi.org/10.1016/j.icheatmasstransfer.2004.04.032.

12. Hunasikatti, S. L.; Suneel, M. P.; Kulkarni, P. S.; Shankar, G. S. S. 2014. Thermal analysis of air flow in a CPU cabinet with motherboard and hard disk as heat sources, International Journal of Mechanical Engineerıng and Technology 5(9) 167-173.

13. Xu, S.; Guo, Z.; Hu, G.; Chen, W.; Lewis, R.; Wong, C.N. 2014. Thermal and flow fields in single board computer cabin systems using CFD analysis, Engineering Applications of Computational Fluid Mechanics 8(4): 574-585. https://doi.org/10.1080/19942060.2014.11083308.

14. Mohan, R.; Govindarajan, P. 2010. Thermal aAnalysis of CPU with composite pin fin heat sinks, International Journal of Engineering Science and Technology 2(9): 4051-4062.
15. Malalasekera, W.; Versteeg, H.K. 2005. An introduction to computational fluid dynamics, The Finite Volume Method, Longman

\section{T. Demircan}

\section{NUMERICAL ANALYSIS OF COOLING DOWN A MICRO ATX COMPUTER CHASSIS}

\section{S u m m a r y}

The technological development in the current environment is in line with the development of computer technologies. Therefore, operation loads of computer processors and capacities and processors and other electronic circuit components are increasing. This increasing load creates over heating on these components. If this high temperature generated on electric circuit components cannot be discarded and safe operating temperatures cannot be obtained, the main components of the computer could get damaged from this high heat and the computer could malfunction. This event presents significant problems as all the system such as health, transportation, and security are dependent on computers to actively operate. In this study Micro ATX computer chassis geometry is designed and cooling the computer chassis with fans is numerically modelled using Computational Fluid Dynamics. Different fan revolutions are calculated to analyze the heat under idle and full-load operations. The simulation results are used for evaluating velocity and temperature distributions in the solution region and operation parameters for optimum cooling are determined.

Keywords: cooling, CPU cooling, computational fluid dynamics (CFD), fluid mechanics.

Received October 10, 2017 Accepted October 18, 2018 